\title{
Transfer Learning Based MA Detection (TL-MAD)
}

\author{
M. Kalpana Devi, M. Mary Shanthi Rani
}

\begin{abstract}
Diabetic Retinopathy (DR) is a microvascular complication of Diabetes that can lead to blindness if it is severe. Microaneurysm (MA) is the initial and main symptom of DR. In this paper, an automatic detection of $D R$ from retinal fundus images of publicly available dataset has been proposed using transfer learning with pre-trained model VGG16 based on Convolutional Neural Network (CNN). Our method achieves improvement in accuracy for $M A$ detection using retinal fundus images in prediction of Diabetic Retinopathy.
\end{abstract}

Keywords : Deep learning (DL), Diabetic Retinopathy (DR), Microaneurysm (MA),Convolutional Neural Networks(CNN)

\section{INTRODUCTION}

$\mathrm{D}_{\text {iabetic Retinopathy (DR) is one of the complicated eye }}$ diseases, commonly affecting diabetic patients. Diabetes affects one in eleven adults around the world.Moreover, huge increase of DR patients is anticipated by 2040. According to World Health Organization (WHO),'DR is the foremost pathology for $4.8 \%$ of the thirty-seven million blindness cases around the world'. Microaneurysm (MA) is observed to be the first stage of DR, a progressive disease and its early stage prediction may protect the vision loss of diabetic people. DR screening has high significance in its prediction. It explores the eye for small red dots and tiny swelling in capillaries of veins. DR screening is normally performed by ophthalmologists through visual inspection of fundus images. But this process is time-consuming, tiresome and highly error prone due to variations in grading by different observers. The alarming rise in the number of DR patients, specifically in under-developed countries calls for effective and automatic approaches for detection of DR.

The process of MA detection poses several challenges as it uses data set of images with different illumination, ill-defined boundaries and varying resolution. Moreover, it may be wrongly interpreted with visually similar structures like haemorrhages, pigmentation patches or dust in lenses etc. Generally, DR detection involves the following four

Revised Manuscript Received on February 15, 2020.

* Correspondence Author

M. Kalpana Devi1, Ph. D., Research Scholar ,Department of Computer Science and Application, Gandhigram Rural Institute(Deemed to be University), Gandhigram, India.

Dr. M. Mary Shanthi Rani, Assistant Professor, Department of Computer Science and Application, Gandhigram Rural Institute (Deemed to be University), Gandhigram, India.

(c) The Authors. Published by Blue Eyes Intelligence Engineering and Sciences Publication (BEIESP). This is an open access article under the CC BY-NC-ND license (http://creativecommons.org/licenses/by-nc-nd/4.0/) processing steps. i) Pre-processing of fundus image. ii) classification of healthy or unhealthy image iii) feature extraction iv) post processing [2]. The progression of DR is categorized into three stages. The earliest stage is named as 'no apparent retinopathy', which describes disappearance of DR in retina. In the second stage MA start to appear which is labelled as Mild DR. Third stage is called severe, with appearance of extra blood vessels in the eye [3]. It uses a set of pretrained weights and achieves performance by fine-tuning hyper parameters [6].

CNN used to extracting important features from the input images. It consist of multiple layers, each layer used to represent the information about the features of an input such as blood vessel structure, leakage of fluids in the form of dots appearance in eye [7].

Deep convolutional neural networks (DCNNs) can be used for end to end process of sourcey medical images to produce an expected outcome prediction [8].Visual Geometrical Group (VGG) is a deep neural network with a multi-layered operation and it is based on CNN Model [10].

Fine-tuning is defined on a CNN with a batch of weights from the weights of a pretrained model. This type of fine-tuning effectively identifies the problem. This method starts with training of fully connected layer and then gradually increasing the network layers during the process until achieving the desired performance [11].Deep learning techniques used in various kind of application such as Plant Disease Detection in Agriculture [13].

The remainder of this paper is organized as follows section II discusses the related research work in this domain, section III describes the proposed work, section IV present the results and discussion and finally conclusion are given in section $\mathrm{V}$.

\section{RELATED WORK}

Piotr Chudzika et al. has explored "Microaneurysm Detection using Fully Convolutional Neural Networks". It contain three steps: preprocessing, patch extraction and pixel-wise classification. A novel patch-based fully convolutional neural network with batch normalization layers and Dice loss function is proposed. Experiments are conducted using datasets with CNN model and the method achieves highest sensitivity at low false positive rates [1].

Mrinal Haloi has developed improved MA detection using deep neural networks.It monitors each pixel of an image and classifies as MA (or) Non-MA using drop out training procedure and maxout activation function. The method is evaluated in publicly available Retinopathy Online Challenge (ROC) and Diaretdb1v2 database to achieve better accuracy [2]. 
Misgina Tsighe Hagos et al. have proposed a novel method for detection of DR based on transfer learning in medical data classification problems. Deep convolutional network pre-trained Inception-V3 model is used in detection of DR.

Stochastic Gradient Descent (SGD) is used to train the input and softmax classifier is used to extract the features. This model resulted in an accuracy of $90.9 \%$ with image augmentation [ 3].

Qiongyao Liang et al. has presented DR detection based on deep learning in five stages No DR, Mild DR, Moderate DR, Severe DR, Proliferative DR. They developed improved InceptionV3 model named multi-self-attention network structure for extraction of features from an input image. This gives higher accuracy (86.7\%) with the updation of weights compared with other pre-trained models like Inception V3 [4]. Muhammad Mateen Junhao Wen et al. has developed an automated medical image analysis technology in DR detection. Combination of a Gaussian mixture model (GMM) and VGGNet are used to extract features. The proposed VGG-19 based model and spatial invariant feature transform (SIFT) to get better classification accuracy (98.13\%) in minimum computation time [7].

Arka Bhowmik et al. has explored detection of various age-related eye abnormalities like Choroidal Neovascularization. They used transfer learning with VGG16 and Inception V3 models to predict the disease. It achieves an improved accuracy of $94 \%$ on the testing data and $99.94 \%$ on training dataset with large amount of data [8].

Sarfaraz Masood et al. has developed a method to identify DR in eye image using deep learning. The data was collected from small datasets. The method used Inception v3 network model with loss function. The features of an input has been identified and remove the imbalance of input data which improves the accuracy of an image [11].

\section{MATERIALS AND METHODS}

In the proposed work, smaller DR datasets have been processed and analysed by using transfer learning with the pre-trained Model (VGG-19).

\section{A. Datasets}

The proposed work was carried out by using three datasets named i) E-Optha ii) ROC iii) Dirat DB1.

E-Ophtha dataset contains 381 compressed images of which 148 have MAs and 233 images are normal healthy fundus images. Images were acquired from 30 screening centres in France at various resolution at 45 FOV (Field of view).

ROC dataset is having fifty training images and fifty test compressed images. Images were captured by three different fundus cameras at various resolutions ranging from $768 \times 576$ to $1389 \times 1383$ at $45^{\circ} \mathrm{FOV}$. All images were labelled with disease levels by experts. In this dataset, 37 images contain MA and remaining 13 images are healthy [1].

DIARETDB1 dataset contains 28 training and 61 test uncompressed images acquired at 50॰ FOV. Each $1500 \times$ 1152 image was manually annotated for presence of MAs and HEs by four medical experts. FPs have no MAs present whereas remaining 51 FPs have at least one MA [2].

\section{B. Methodology}

The Proposed Methodology involves three major steps:

i. Pre-processing: The main objective of pre-processing is to resize the image into $(224 * 224)$, in order to improve the resolution of an image. Pre-processing is helpful and it is used to distinguish between the normal and Microaneurysm (MA) affected images. The input images were resized to 224x224 dimension by using the opencv2 in Python.

ii. Training with CNN: Simonyan and Zisserman from Visual Geometry Group (VGG) designed the network model VGG19 with sixteen layers using a set of (3x3) convolution filters for feature extraction. It is mostly suited for transfer learning [6]. This model contains max pooling layers to reduce the dimensionality and is trained with ImageNet dataset [8].

ImageNet is a dataset which contains fifteen million labelled high-resolution natural images belonging to approximately twenty two thousand categories. It is experimented and it is also called the ImageNet Large-Scale Visual Recognition Challenge (ILSVRC).All pretrained models are trained using the images on ImageNet dataset and it is also publicly available [14].VGGNet out performs Alex Net Model because of its simple architecture [12].

Transfer learning is defined as the reuse of pre-trained DNN models to classify the data on large datasets. By using the transfer learning technique, weights of a network are trained on a source database and the result is evaluated with the destination database [5]. Combining with pre-trained model gives better extraction of low level features, with higher value of training and testing accuracy [6]. It obtains the initial weights from the pre-trained model and extracts features like colour and size from a training set by updating the weights with optimal values for prediction [11].

iii. Validation and Testing the trained model: The trained model is validated and tested using small DR sets E-optha, ROC and DIRAT DB1 based on performance metrics Accuracy, Precision, Recall and F1-Score.

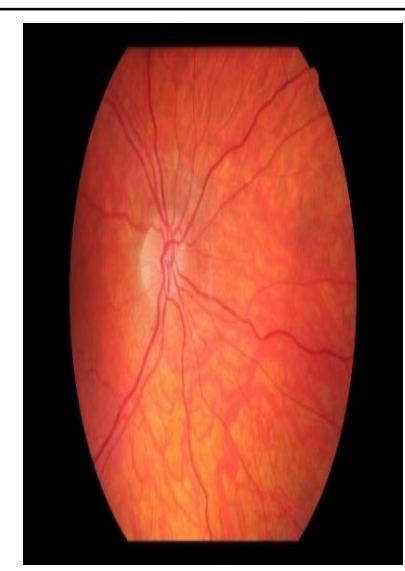

(a)

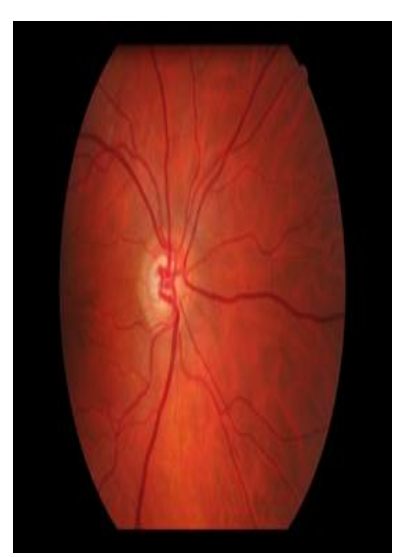

(b)

\section{Fig.1. a) Healthy image image \\ b) Microaneurysm}

The flow diagram of the proposed method is depicted as follows: 


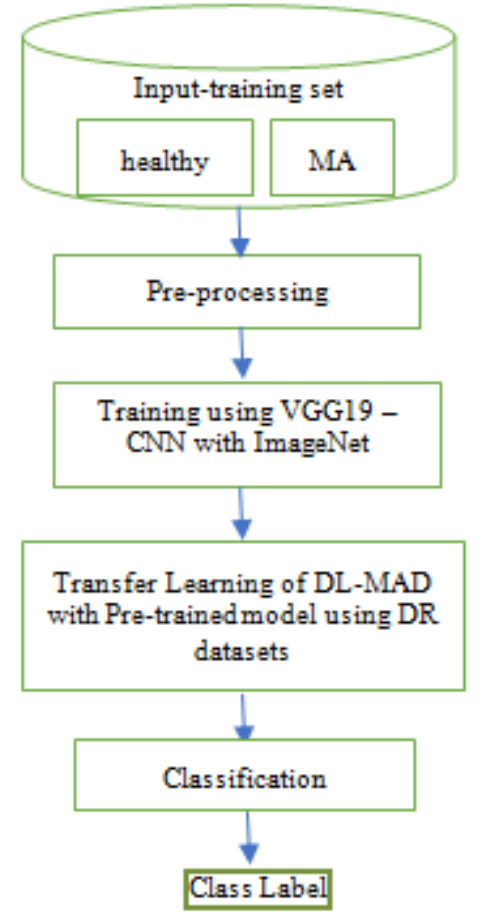

Fig 2. Flow diagram of TL-MAD.

\section{RESULTS AND DISCUSSION}

The proposed TL-DRD model is trained with publicly available dataset for prediction of diabetic retinopathy. The first step is pre-processing stage in which the images have been resized to $224 * 224$ of resolution and stored into NumPy arrays with the dimension of the same value of resolution. Adam optimizer is used for training. Each dataset is divided into training set and test set in the ratio 70:30. Training set is used to train the model and test set is used to validate the model.

Table 1 presents the comparison of performance of the proposed model with similar methods interms of accuracy. The table also lists the datasets used for training and testing.

Table 1: Performance comparison

\begin{tabular}{|c|c|c|c|c|}
\hline $\begin{array}{c}\text { S.n } \\
\text { o }\end{array}$ & $\begin{array}{l}\text { Model } \\
\text { Used }\end{array}$ & Method Used & $\begin{array}{c}\text { Dataset } \\
\text { Used }\end{array}$ & $\begin{array}{c}\text { Accurac } \\
\mathbf{y} \\
(\%) \\
\end{array}$ \\
\hline 1. & $\begin{array}{l}\text { Inception- } \\
\text { V3 }\end{array}$ & $\begin{array}{l}\text { Using Stochastic } \\
\text { Gradient Operator } \\
\text { with deep learning } \\
\text { model[3]. }\end{array}$ & $\begin{array}{l}\text { Kaggle } \\
\text { DR }\end{array}$ & 90.9 \\
\hline 2. & VGG-19 & $\begin{array}{l}\text { Deep Neural Networks } \\
\text { with back propagation } \\
{[6] \text {. }}\end{array}$ & Eyewes & 80.19 \\
\hline \multirow{3}{*}{3.} & \multirow{3}{*}{ VGG-19 } & \multirow{3}{*}{$\begin{array}{l}\text { Proposed model } \\
\text { TL-MAD }\end{array}$} & $\begin{array}{l}\text { Dirat } \\
\text { DB1 }\end{array}$ & 85.2 \\
\hline & & & ROC & 94.5 \\
\hline & & & $\begin{array}{l}\text { E-Opth } \\
\text { a }\end{array}$ & 95.1 \\
\hline
\end{tabular}

It is obvious from Table1, the accuracy has been obtained that high accuracy by TL-MAD, comparable to similar methods.

Fig. 3 shows the graphical representation of the comparison of the performance of the method.

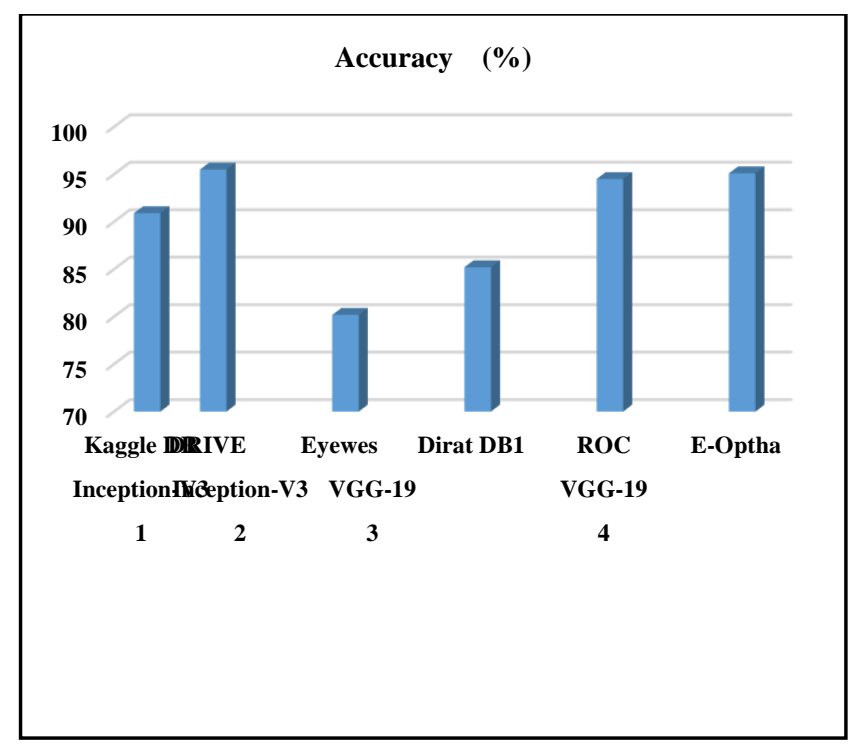

Fig .3. Accuracy comparison of TL-MAD

Experiments were also conducted by freezing the first, third and fifth layers of the proposed TL-MAD model so as to reduce the complexity. Table 2 shows the results interms of accuracy for all the three dataset

Table 2 performance analysis by freezing the layers

\begin{tabular}{|c|c|c|c|}
\hline S.No & Dataset & Convolution layer & $\begin{array}{c}\text { Accuracy (\%) } \\
\text { (Using } \\
\text { pretrained-VGG19 } \\
\text { Model) }\end{array}$ \\
\hline \multirow{3}{*}{1.} & \multirow{3}{*}{ Dirat-DB1 } & Freeze $1^{\text {st }}$ layer & 70 \\
\hline & & Freeze 3rd layer & 75 \\
\hline & & Freeze $5^{\text {th }}$ layer & 81.76 \\
\hline \multirow{3}{*}{2.} & \multirow{3}{*}{ E-Optha } & Freeze $1^{\text {st }}$ layer & 75.1 \\
\hline & & Freeze 3rd layer & 82.7 \\
\hline & & Freeze $5^{\text {th }}$ layer & 94.15 \\
\hline \multirow{3}{*}{3.} & \multirow{3}{*}{ ROC } & Freeze $1^{\text {st }}$ layer & 81.17 \\
\hline & & Freeze $3^{\text {rd }}$ layer & 90.23 \\
\hline & & Freeze $5^{\text {th }}$ layer & 95.1 \\
\hline
\end{tabular}

It is evident from the Table2 that high accuracy is achieved when freezing.

Fig 4 shows the pictorial representation of the performance of TL-MAD with different by freezing the layers.

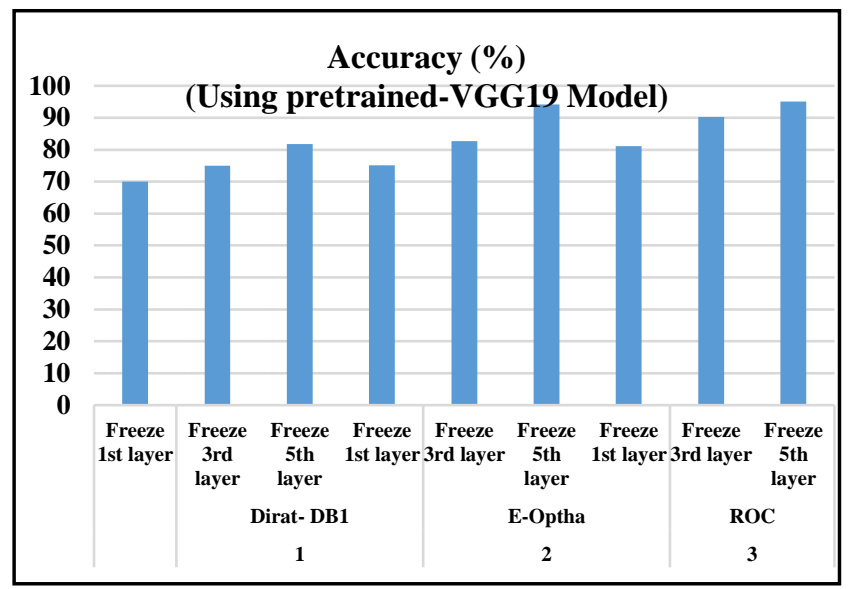

Fig 4. Accuracy of TL-MAD with freezing process 
The performance of the proposed TL-MAD model interms of precision, Recall and F1-Score is demonstrated in Table 3.

Table 3: Performance Metrics

\begin{tabular}{|c|c|c|c|c|}
\hline S.No & Dataset & Precision & Recall & F1-Score \\
\hline 1 & Dirat DB1 & 0.77 & 1.00 & 0.87 \\
\hline 2 & ROC & 0.88 & 0.93 & 0.91 \\
\hline 3 & E-Optha & 0.98 & 0.98 & 0.98 \\
\hline
\end{tabular}

Table 3 clearly demonstrate the superior performance of TL-MAD model for e-optha data set and the graphical representation is shown in Fig 5.

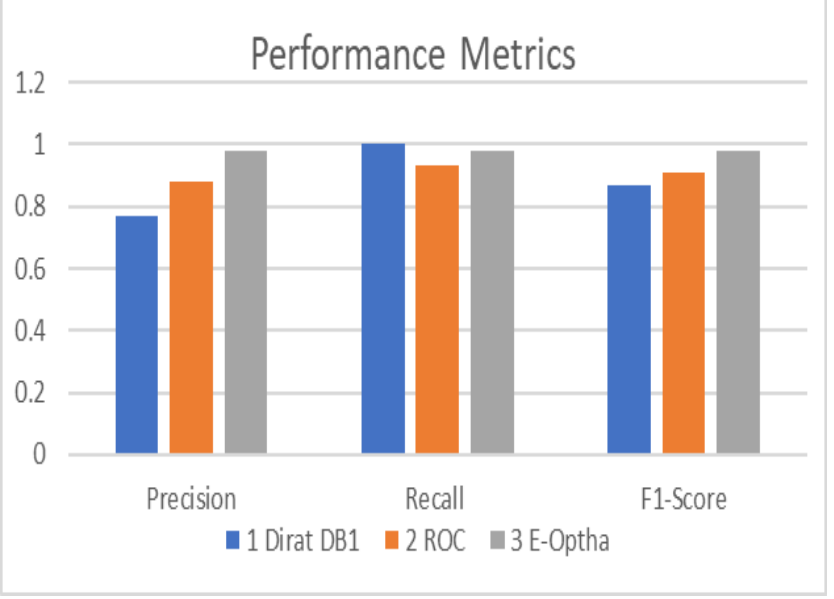

Fig 5 Performance Analysis using classification metrics

\section{CONCLUSION}

In this paper, transfer learning is used to classify DR images into two classes with three different small data sets. We design the proposed TL-DRD model whose weights are initialized from a pre-trained CNN model. The model is also Experimented by freezing the layers to reduce the complexity of the network. Results have endorsed the superiority of the proposed model over existing model, interms of enhanced accuracy.

\section{REFERENCES}

1. Chudzik, Piotr, Somshubra Majumdar, Francesco Calivá, Bashir Al-Diri, and Andrew Hunter. "Microaneurysm detection using fully convolutional neural networks." Computer methods and programs in biomedicine 158 (2018): 185-192.

2. Haloi, Mrinal. "Improved microaneurysm detection using deep neural networks." arXiv preprint arXiv:1505.04424 (2015).

3. Hagos, Misgina Tsighe, and Shri Kant. "Transfer Learning based Detection of Diabetic Retinopathy from Small Dataset." arXiv preprint arXiv:1905.07203 (2019).

4. Liang, Qiongyao, Xiangkui $\mathrm{Li}$, and Yansong Deng. "Diabetic Retinopathy Detection Based on Deep Learning." (2019).

5. Gelman, Rony. "Evaluation of Transfer Learning for Classification of:(1) Diabetic Retinopathy by Digital Fundus Photography and (2) Diabetic Macular Edema, Choroidal Neovascularization and Drusen by Optical Coherence Tomography." arXiv preprint arXiv:1902.04151 (2019).

6. Costa, Pedro, Teresa Araújo, Guilherme Aresta, Adrian Galdran, Ana Maria Mendonça, Asim Smailagic, and Aurélio Campilho. "EyeWes: weakly supervised pre-trained convolutional neural networks for diabetic retinopathy detection." In 2019 16th International Conference on Machine Vision Applications (MVA), pp. 1-6. IEEE, 2019.

7. Mateen, M., Wen, J., Song, S., \& Huang, Z. (2019). Fundus image classification using VGG-19 architecture with PCA and SVD. Symmetry, 11(1), 1

8. Masood, S., Luthra, T., Sundriyal, H., \& Ahmed, M. (2017, May). Identification of diabetic retinopathy in eye images using transfer learning. In 2017 International Conference on Computing, Communication and Automation (ICCCA) (pp. 1183-1187). IEEE.Learning

9. Alban, Marco, and Tanner Gilligan. "Automated detection of diabetic retinopathy using fluorescein angiography photographs." Report of standford education (2016).

10. Bhowmik, Arka, Sanjay Kumar, and Neeraj Bhat. "Eye Disease Prediction from Optical Coherence Tomography Images with Transfer Learning." In International Conference on Engineering Applications of Neural Networks, pp. 104-114. Springer, Cham, 2019.

11. Li, X., Pang, T., Xiong, B., Liu, W., Liang, P., \& Wang, T. (2017, October). Convolutional neural networks based transfer learning for diabetic retinopathy fundus image classification. In 2017 10th International Congress on Image and Signal Processing, BioMedical Engineering and Informatics (CISP-BMEI) (pp. 1-11). IEEE.

12. Wan, Shaohua, Yan Liang, and Yin Zhang. "Deep convolutional neural networks for diabetic retinopathy detection by image classification." Computers \& Electrical Engineering 72 (2018) 274-282.

13. Sangeetha R, Mary Shanthi Rani M, "Tomato leaf disease prediction using convolutional neural networks" International Journal of Innovative Technology and Exploring Engineering (IJITEE) ISSN: 2278-3075, Volume-9 Issue-1, pp 1348-1352,November 2019

\section{AUTHORS PROFILE}

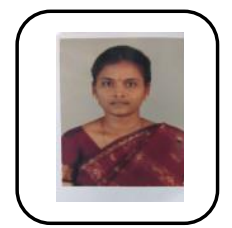

Image Processing.
M. Kalpana Devi, Received B.Sc degree in physics from Gandhigram Rural Institute(Deemed to be university), Gandhigram, M.C.A from Navarasam Arts \& Science College, Bharathiyar University, Joined as a Research Scholar, Gandhigram Rural Institute (Deemed to be university). Interested in development of Deep learning Techniques in medical

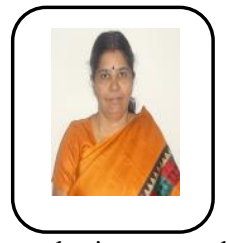

Dr. M. Mary Shanthi Rani, holds Ph.D in Computer Science and has more than 14 years of teaching experience .She has great passion for teaching and is currently working as Assistan Professor in the Department of Computer Science and Applications, The Gandhigram Rural Institute (Deemed to be University), Gandhigram. She has nearly sixty two publications in International Journals and Conferences .Her research areas of interest are Image Compression, Information Security, Ontology, Biometrics and Computational Biology and Deep Learning. She has served in various academic committees in designing the curriculum for B.Sc. and M.C.A courses as well. She has also served as reviewer of Peer-reviewed International Journals and Conferences. She is a Life member of Indian Society for Technical Education. She has the credit of being the Associate Project Director of UGC Indo-US 21st Knowledge Initiative Project. She has the credit of being the Associate Project Director of Pandit Madan Mohan Malaviya National Mission n Teachers and Teaching (PMMMNMTT).She has a Lead zonal partner of LeadingIndia.ai. 